\title{
LATTICE RELAXATION AROUND ARSENIC AND SELENIUM IN CdTe
}

\author{
V. Koteski ${ }^{1}$, H. Haas, E. Holub-Krappe, N. Ivanovic ${ }^{1}$, and H.-E. Mahnke ${ }^{*}$ \\ Hahn-Meitner-Institut Berlin GmbH, Bereich Strukturforschung \\ D-14109 Berlin, Germany
}

\begin{abstract}
We have investigated the lattice relaxation around impurity atoms at the anion sublattice in $\mathrm{CdTe}$, such as As acting as acceptor and Se which is isovalent to Te, with fluorescence detected EXAFS. We experimentally verify the lattice relaxation with a bond length being reduced by $8 \%$ around the As atom as inferred indirectly from ab-initio calculations of the electric field gradient in comparison with the measured value in a PAC experiment (S. Lany et al., Phys. Rev. B 62, R2259 (2000)). In the case of the isovalent impurity atom Se, the bond length is similarly reduced as determined experimentally by EXAFS and by model calculations with the density functional theory implemented in the WIEN97 program. In contrast to this inward relaxation, preliminary calculations for $\mathrm{Br}$ in $\mathrm{CdTe}$, the next element in the series $\mathrm{As}, \mathrm{Se}$, and $\mathrm{Br}$, which acts as donor at the Te sublattice, indicate an increase in bond length, an interesting prediction waiting for experimental verification.
\end{abstract}

PACS codes: 61.72.Ji, 61.72.Vv, 61.10.Ht , 71.15.Mb , 71.55.Gs

\section{Introduction.}

The incorporation of impurity atoms is often accompanied with lattice distortions and the formation of defect complexes. Local strain fields introduced by the impurities can drastically influence electrical and optical properties. Knowledge about these local effects is mainly derived from measurements, which determine the local structure only indirectly ( like e.g. Electron Spin Resonance, Perturbed Angular Correlation (PAC), Zeeman- and Piezoscopic FTIR studies). A rather direct experimental access to lattice distortions is provided by the Xray absorption spectroscopy (EXAFS). In this contribution we report on measurements of the lattice relaxation around the acceptor dopant $\mathrm{As}$, and the isovalent impurity $\mathrm{Se}$ at the $\mathrm{Te}$ sublattice in CdTe with EXAFS. The experimental challenge lies in the necessary compromise between a concentration high enough for an EXAFS detection, but low enough to avoid compensation and clustering of the dopants in the semiconductors. In the case of As

\footnotetext{
*Corresponding author: E-mail: mahnke@hmi.de

${ }^{1}$ Permanent address: VINČA Institute of Nuclear Science, POB 522, 11001 Belgrade,Yugoslavia
} 
in $\mathrm{CdTe}$, the aim was to experimentally verify the lattice relaxation around the impurity atom as determined theoretically with the LAPW method used to calculate the electric field gradient in comparison with the measured value in a PAC experiment [1]. In order to understand the driving force for the lattice relaxation we also determined the relaxation around Se, the neighbouring element to As and isovalent to Te, for which no doping effects in $\mathrm{CdTe}$ are known. Finally we have extended our calculation to the next neighbouring element, $\mathrm{Br}$, acting as donor when incorporated substitionally at the Te sublattice.

\section{Experiment and theory.}

\subsection{Experimental details.}

The incorporation of arsenic as the acceptor dopant in CdTe was done by ion implantation at the tandetron of FZ Rossendorf. A total dose of $7 \times 10^{15} \mathrm{~cm}^{-2}$ As atoms was almost uniformly distributed up to a depth of $3 \mu \mathrm{m}$. The samples, originally from Crystec $\mathrm{GmbH}^{2}$, were thermally treated before and after the implantation following the approaches [2] in which As was predominantly incorporated as acceptor at the Te site as checked by photoluminescence (PL) measurements. To reduce background in the fluorescence detected EXAFS from undoped sample material, the crystals were thinned from the back to a total thickness of about $30 \mu \mathrm{m}$. For the case of Se in CdTe we used mixed CdTe $\mathrm{Cl}_{-\mathrm{x}} \mathrm{Se}_{\mathrm{x}}$ crystals, provided by M. Fiederle $^{3}$, which were powdered, mixed with graphite or polyethylene, and pressed into pellets.

To determine the local structure around As and Se we have measured the K-edge absorption at the X1-beamline of HASYLAB at DESY. In the case of As we measured the absorption in the fluorescence mode with the 5-segment Ge-detector, the fluorescence being detected in line with the polarisation vector of the incoming synchrotron radiation. To reduce the influence from elastic and inelastic scattering of the incoming radiation, critical absorption was employed using a foil made out of Ge powder mixed with polyethylene. In the case of Se, the absorption could be measured in transmission on the powdered samples. The absorption on $\mathrm{Cd}$ and Te was measured in transmission, too. For the measurements, the samples were mounted on a He gas flow cryostat, keeping the temperature at approximately 18 or $300 \mathrm{~K}$.

The analysis of the observed absorption spectra was done following the standard FEFF procedure [3, 4] including the background treatment with AUTOBK [5]. In the case of Se in the mixed crystal, a combined multiple data set fitting procedure of the data recorded for Se as well as for $\mathrm{Cd}$ and $\mathrm{Te}$ data could be applied.

\subsection{Theoretical details.}

Complementing our experimental work we have performed ab-initio calculations based on the density functional theory (DFT) with the WIEN97 package [6] which uses the linearised augmented plane wave (LAPW) method and with the FHI96md program [7] which uses firstprinciples pseudo-potentials (PP) and a plane-wave basis set. The latter was used to investigate the size dependence of the super-cells constructed around one substitutional As atom in CdTe. The calculations yielded good agreement with our EXAFS experiment (see Table1) so that the determined relaxations can be used as a solid basis for further interpretations of derived parameters such as hyperfine interaction parameters of defect complexes (for further details see $[1,8]$ ).

\footnotetext{
${ }^{2}$ Crystec GmbH, D-12555 Berlin

${ }^{3}$ M. Fiederle, Freiburger Materialforschungszentrum, private communication
} 


\section{Results and discussion.}

An example of our EXAFS result at low temperature is given in Fig.1. As checked for two different samples, the main features of the absorption spectra at $20 \mathrm{~K}$ and at room temperature remain the same. Since our main concern was that the concentration may be too close to the doping limit, we checked the uniqueness of the As configuration. The mentioned temperature behaviour may be taken as a first, although weak proof for the structural uniqueness of the As configuration. Similarly, the detected PL corresponding to the As acceptor line in CdTe may not be a sufficient proof for the occurrence of the As-on-Te-site configuration as the main or sole As configuration either. As an additional test, we have inspected the XANES region of the absorption spectra and compared it to model spectra assuming As in various configurations (see Fig.2). The modelling was performed according to [9]. Only the description of As being substitutionally at the Te site matches the experimental spectra. The occurrence of a ,white line“ in our experiment allows to safely state that the contribution from other As configurations cannot exceed a few percent of the total As concentration. We can therefore adopt the determined bond length as representative for As in CdTe at the substitional Te site.

In Tab. 1 we compare our measured bond length with the results obtained from the pseudopotential calculations as well as the LAPW values according to ref. [1]. The agreement is very good. A distinction between the two possible impurity charge states cannot be made, the difference is too small to be noticeable in our data taken our experimental accuracy into account. There is, however, a tendency that the bond length extracted from our XAFS data is shorter by approximately $0.04 \AA$ at room temperature than at $20 \mathrm{~K}$ which could be the result of a change of the charge state, or at least of a shift in the occupation of the possible impurity charge states.

Our EXAFS data on Se and the corresponding calculated values can be considered as an additional support to the good agreement obtained for As in CdTe between experiment and model calculation. The experimental results for Se in $\mathrm{CdTe}_{1-\mathrm{x}} \mathrm{Se}_{\mathrm{x}}$ are illustrated in Fig.3. The extracted values are included in Tab.1. We have chosen a concentration of $x=0.04$ which comes close to the super-cell size of 32 atoms. The agreement is very good and gives confidence to attack more sophisticated configurational doping problems both by modelling and the experimental verification of proposed model description. In this context we present our result of bond length calculation for the donor $\mathrm{Br}$ in CdTe, included in the Tab.1. Contrary to the As and Se case, it shows a sizeable increase in bond length. This prediction is awaiting experimental verification, which would probably help us to understand the formation of $\mathrm{Br} \mathrm{A}-$ centre in CdTe.

\section{Acknowledgement}

The authors are grateful to the HASYLAB staff at DESY, in particular to N. Haack and E. Welter. We thank H. Rossner for his advice on analysis and fitting of the data, for P. Szimkowiak for his help in sample preparation as well as M. Fiederle from the Freiburger Materialforschungszentrum for the $\mathrm{CdTe}_{1-\mathrm{x}} \mathrm{Se}_{\mathrm{x}}$ sample. Help in sample preparation is also acknowledged for the As implantation by M. Friedrich (FZ Rossendorf), and crystal thinning and PL tests by S. Lany, F. Wagner and H. Wolf (University Saarbrücken). We further thank J. Bollmann and J. Weber from the TU Dresden and S. Lany and T.Wichert from the U Saarbrücken for fruitful discussions on the problem of lattice relaxation around dopants in semiconductors. Finally, we gratefully acknowledge the help of G. Schwarz from the FHI Berlin in setting up the input parameters for the FHI96md computer code. 


\section{References}

[1] S. Lany et al., Phys. Rev. B62, R2259 (2000)

[2] e.g. S. Lany et al., Physica B302-303, 114 (2001) and ref. therein.

[3] J.J. Rehr, J. Mustre de Leon, S.I. Zabinsky, and R.C. Albers, J. Am. Chem. Soc.113, 5135 (1991)

[4] E.A. Stern, M. Newville, B. Ravel, Y. Yacoby, and D. Haskel, Physica B208\&209, $117(1995)$

[5] M. Newville, P. Livins, Y.Yacoby, J.J. Rehr, and E.A. Stern, Phys. Rev. B47, 14126 (1993)

[6] P. Blaha, K. Schwarz, J.Luitz, WIEN97, A Full Potential Linearised Augmented Plane Wave Package for Calculating Crystal Properties, (Karlheinz Schwarz, TU Wien, Austria, 1999), ISBN 3-9501031-0-4.

[7] M. Bockstedte, A. Kley, J. Neugebauer and M. Scheffler, Comp. Phys. Comm. 107, 187 (1997)

[8] V. Koteski, N. Ivanovic, H. Haas, E. Holub-Krappe, H.-E. Mahnke, NIM B200, 60 (2003)

[9] A. Ankudinov, B. Ravel, J.J. Rehr, S. Conradson, Phys. Rev. B 58, 7565 (1998) 


\section{Table 1}

Comparison of experimentally determined nearest neighbour and next-nearest neighbour distances around impurity (dopant) atoms in CdTe with calculated values obtained with LAPW (super-cell size 32 atoms) and with PP methods (super-cell size up to 216 atoms). The calculated values are given for the charged configuration in the case of As and Br. Distances for the neutral configuration are closer to the undoped material values by $0.01-0.02 \AA$ in the case of As and approximately $0.04 \AA$ in the case of $\mathrm{Br}$. (All values are given in $\AA$ ).

\begin{tabular}{|c|c|c|c|c|c|c|}
\hline \multirow[t]{2}{*}{ System } & \multirow[t]{2}{*}{$\mathrm{R}_{\mathrm{nn}}(\exp )$} & \multicolumn{2}{|c|}{$\mathrm{R}_{\mathrm{nn}}($ theo) } & \multirow[t]{2}{*}{$\mathrm{R}_{\mathrm{nnn}}(\exp )$} & \multicolumn{2}{|c|}{$\mathrm{R}_{\mathrm{nnn}}($ theo) } \\
\hline & & LAPY & $\mathrm{PP}$ & & LAPW & PP \\
\hline CdTe & $2.806^{*}$ & & & $4.583 *$ & & \\
\hline $\mathrm{As}$ in $\mathrm{CdTe}$ & $2.58(2)$ & $2.57^{\#}$ & 2.57 & $4.50(6)$ & $4.52^{\#}$ & 4.55 \\
\hline Se in CdTe & $2.67(1)$ & 2.66 & & $4.53(1)$ & 4.55 & \\
\hline $\mathrm{Br}$ in $\mathrm{CdTe}$ & $?$ & 2.90 & & $?$ & 4.57 & \\
\hline
\end{tabular}

*Values derived from the standard lattice parameter

${ }^{\#}$ Values as presented in ref. [1]

\section{Figure Captions}

Figure 1: Radial distribution function of As in CdTe obtained from the EXAFS spectrum, weighted linearly with $\mathrm{k}$ and Fourier transformed. The fit region in R-space is indicated by the bar. The insert shows the EXAFS spectrum $\chi(\mathrm{k})$.

Figure 2: Comparison of the experimental XAFS signal with XANES spectra calculated for different configurations around As in CdTe: (i) As substitutionally on the Te site $\left(\mathrm{As}_{\mathrm{Te}}\right)$, (ii) As on the substitutional $\mathrm{Cd}$ site $\left(\mathrm{As}_{\mathrm{Cd}}\right)$, and As located interstitially with (iii) either $\mathrm{Te}\left(\mathrm{As} \mathrm{s}_{\mathrm{int} 1}\right)$ or (iiii) $\mathrm{Cd}\left(\mathrm{As}_{\mathrm{int} 2}\right)$ as first-shell neighbours. The theoretical spectra are normalised to the experimental edge step and shifted in y-direction for easier comparison.

Figure 3: EXAFS spectrum $\chi(\mathrm{k})$ (top) and radial distribution function (bottom) of Se in $\mathrm{CdTe}_{1-\mathrm{x}} \mathrm{Se}_{\mathrm{x}}$ for $\mathrm{x}=0.04$, obtained from the EXAFS spectrum, weighted linearly with $\mathrm{k}$ and Fourier transformed. The fitting range in R-space runs from 1.9 to $5 \AA$. 


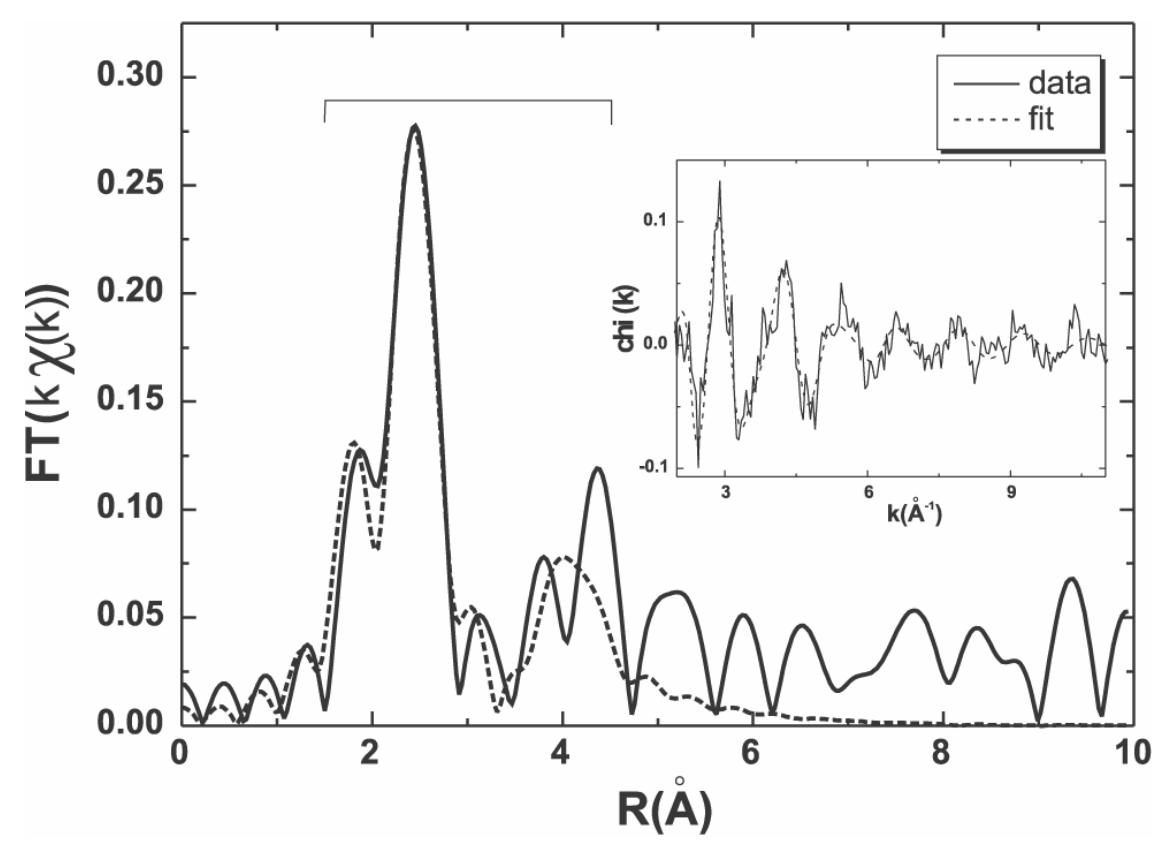

Fig. 1

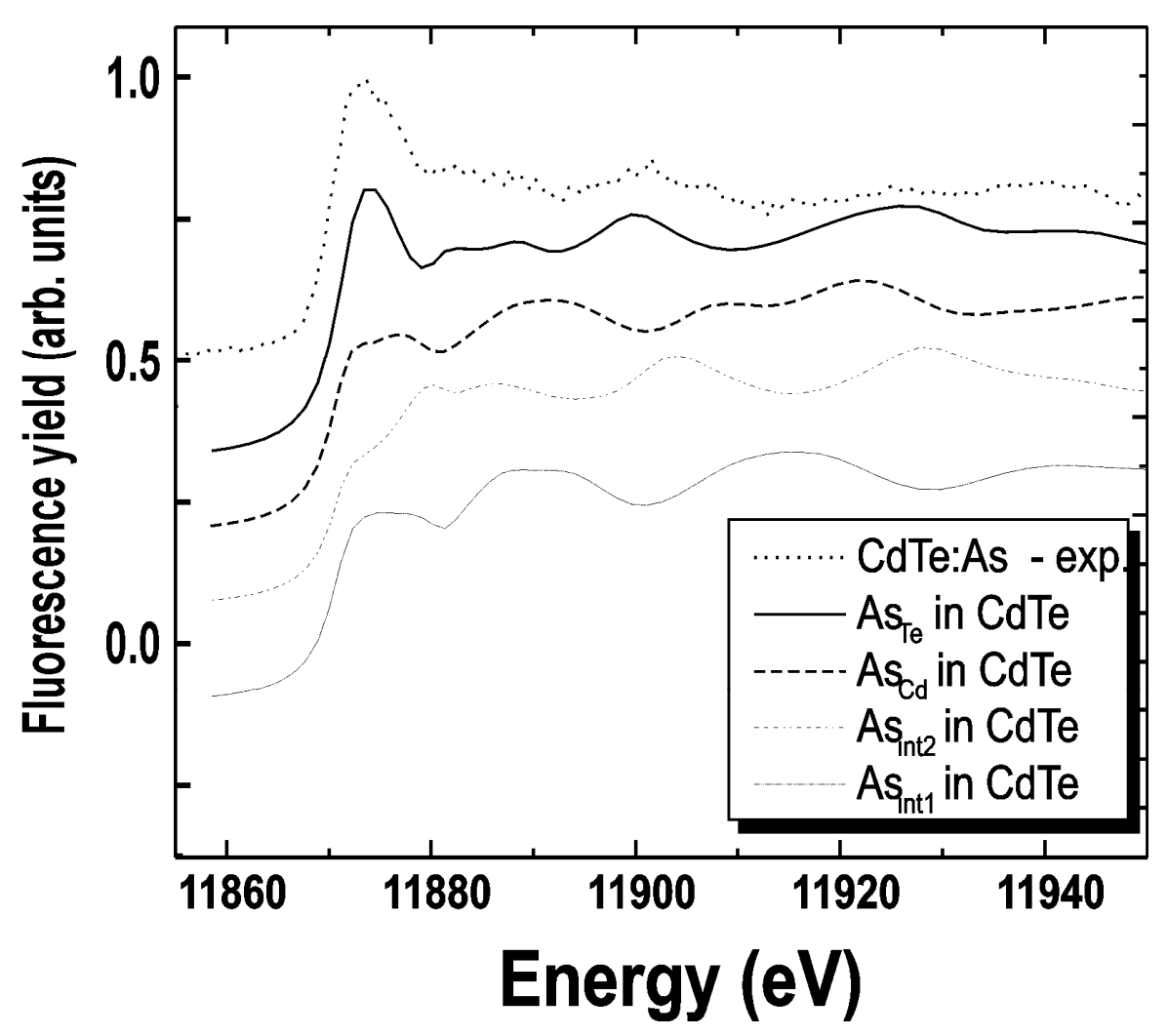

Fig. 2 

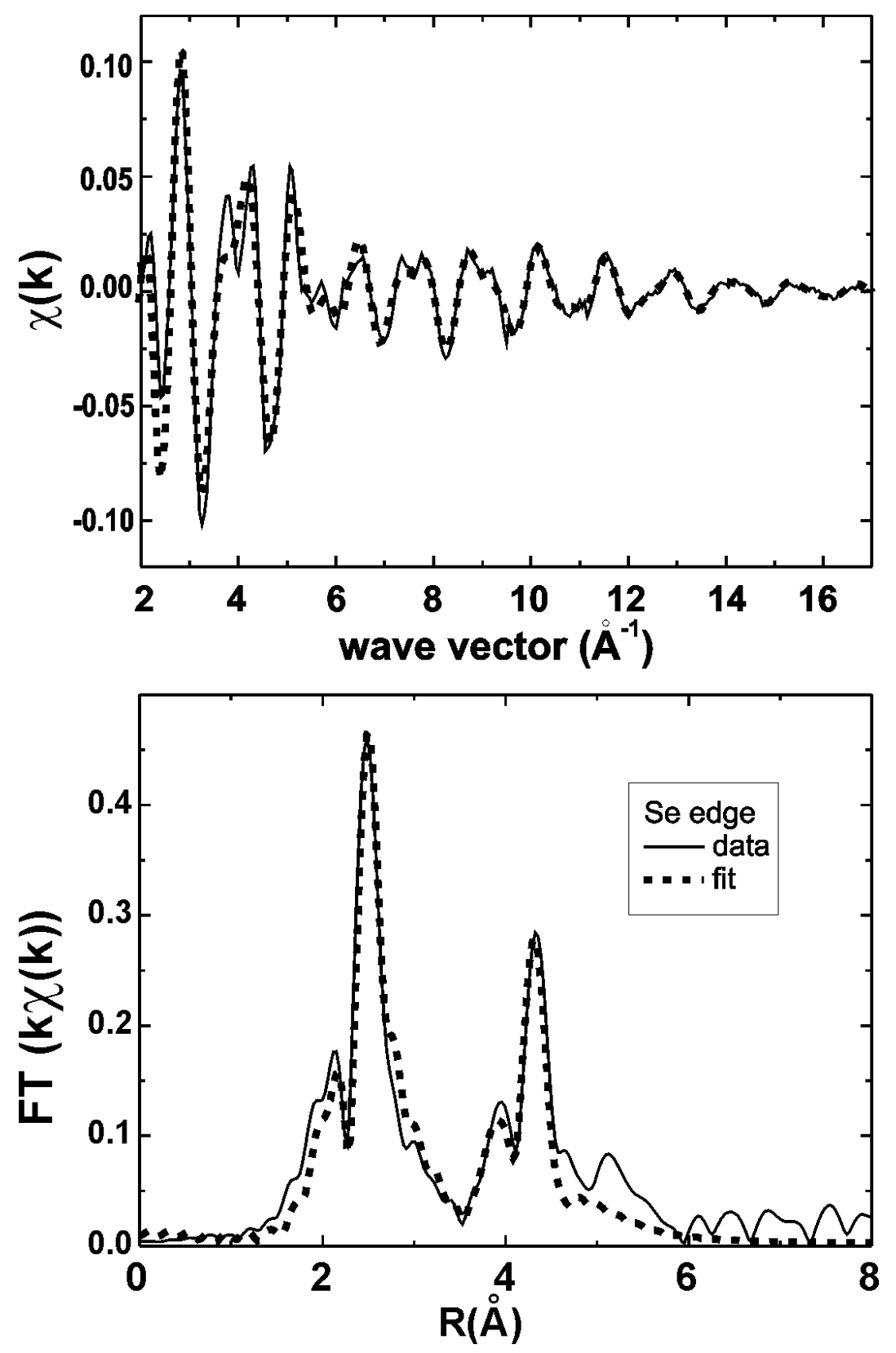

Fig. 3 